\title{
Ectopic fat, insulin resistance and metabolic disease in non-obese Asians: investigating metabolic gradation
}

\author{
Yoshifumi Tamura ${ }^{1), 2), 3)}$ \\ 1) Department of Metabolism \& Endocrinology, Juntendo University Graduate School of Medicine, Tokyo, Japan \\ 2) Sportology Center, Juntendo University Graduate School of Medicine, Tokyo, Japan \\ 3) Faculty of International Liberal Arts, Juntendo University, Tokyo, Japan
}

\begin{abstract}
Although metabolic abnormalities commonly occur in non-obese Asians, their pathogenesis is not fully understood. Proton magnetic resonance spectroscopy has been used to analyze intracellular lipids in humans, and results suggest that ectopic fat accumulation in muscle and liver may induce insulin resistance in each tissue independently of obesity. Thus, measurement of ectopic fat currently plays an important role in the study of insulin resistance in non-obese Asians. In addition, studies using 2-step hyperinsulinemic euglycemic clamp with a glucose tracer may clarify how tissuespecific insulin resistance in muscle, liver, and adipose tissue contributes to the development of metabolic disease in nonobese Japanese. Although numerous studies have elucidated the pathophysiology of insulin resistance in obese subjects, research on "metabolic gradation," defined as the gradual transition from an insulin-sensitive to an insulin-resistant state, is less common, especially in terms of early metabolic changes. This review addresses a simple question: when and how is insulin resistance induced in non-obese East Asians? Several studies revealed that impaired insulin clearance and hyperinsulinemia not only compensated for insulin resistance, but also secondarily facilitated insulin resistance and weight gain. In this regard, we recently found that impaired insulin clearance and hyperinsulinemia could occur in apparently healthy subjects without significant insulin resistance, suggesting that this change may be an initial trigger that drives subsequent insulin resistance and weight gain. Further research is required to clarify the pathogenesis of metabolic gradation in non-obese Asians.
\end{abstract}

Key words: Insulin resistance, Intramyocellular lipid, Intrahepatic lipid, Physical activity, Non-obese East Asian

\section{Introduction}

Insulin resistance, which occurs more commonly in those with increased body weight, is known to play an important role in metabolic abnormalities in obesity [1]. Many previous studies have suggested that elevation of free fatty acids (FFAs) is one of the mechanisms of insulin resistance in obese subjects. In fact, elevated circulating FFA concentrations are observed in insulin-resistant obese subjects [2], and artificial FFA elevation by lipid infusion induces insulin resistance in muscle and liver in healthy individuals $[3,4]$. On the other hand, proton magnetic resonance spectroscopy ( $\left.{ }^{1} \mathrm{H}-\mathrm{MRS}\right)$ has been used to analyze intracellular lipids in humans, and has shown that ectopic fat accumulation in muscle and liver

Submitted Oct. 5, 2018; Accepted Nov. 6, 2018 as EJ18-0435 Released online in J-STAGE as advance publication Dec. 4, 2018 Correspondence to: Yoshifumi Tamura, MD, PhD, Department of Metabolism \& Endocrinology, Juntendo University Graduate School of Medicine, 2-1-1 Hongo, Bunkyo-ku, Tokyo 113-8421, Japan.

E-mail: ys-tamur@juntendo.ac.jp contributes to insulin resistance in each tissue [5]. Circulating FFAs have been found to be an important source of ectopic fat accumulation in muscle [4] and liver [6], and thus FFAs released by adipose tissue in obese individuals may accumulate as ectopic fat, serving as a downstream mechanism of insulin resistance.

Even non-obese Asians $\left(<25 \mathrm{~kg} / \mathrm{m}^{2}\right)$ readily develop metabolic abnormalities [7-10]. The World Health Organization has suggested that in Asians, overweight as a body mass index (BMI) $\geq 23 \mathrm{~kg} / \mathrm{m}^{2}$, and obesity as BMI $\geq 25 \mathrm{~kg} / \mathrm{m}^{2}$ [10], although in other ethnicities these conditions are defined as BMI $\geq 25 \mathrm{~kg} / \mathrm{m}^{2}$ and $\mathrm{BMI} \geq 30 \mathrm{~kg} / \mathrm{m}^{2}$, respectively. Insulin resistance is often recognized in obese individuals worldwide, but in non-obese Asians its etiology is not fully understood and it is unclear whether elevated FFAs and ectopic fat accumulation play an important pathogenic role in this population. Thus, this review addresses the results of research on ectopic fat over the past 2 decades, then discusses hypotheses regarding the mechanisms of insulin resistance in nonobese Asians. 


\section{Measurement of Insulin Sensitivity in Adipose Tissue, Muscle and Liver}

Insulin has several metabolic effects on target organs. For example, it increases glucose uptake in skeletal muscle and suppresses hepatic glucose production. It also has an anti-lipolytic effect in adipose tissue and decreases the level of circulating FFAs. Hyperinsulinemic euglycemic glucose clamp with a glucose tracer is the gold standard for evaluating tissue-specific insulin sensitivity in insulin target organs [11]. A glucose tracer (e.g., $\left[6,6-{ }^{2} \mathrm{H}_{2}\right]$ glucose) is necessary to evaluate endogenous glucose production (EGP) [12]. Peripheral insulin sensitivity is evaluated by the insulin-stimulated rate of disappearance (Rd) of glucose. If glucose clamp is performed without a glucose tracer but with a high insulin infusion rate $(\sim 100$ $\mathrm{mU} / \mathrm{m}^{2}$ per $\mathrm{min}$ ), the glucose infusion rate (GIR) to maintain euglycemia indicates peripheral glucose uptake, because EGP is completely suppressed by insulin [11]. Because skeletal muscle is the major tissue involved in peripheral glucose uptake during glucose clamp, Rd and GIR are used as markers of muscle insulin sensitivity. However, if the insulin infusion rate is somewhat lower (e.g., $40 \mathrm{mU} / \mathrm{m}^{2}$ per min), EGP may not be completely suppressed [11], and thus GIR should be used as a marker for whole-body insulin sensitivity. The metabolic clearance rate of glucose (MCR), a similar index to GIR, is sometimes used as marker of whole-body insulin sensitivity $[13,14]$. In addition, insulin-mediated suppression of EGP can be measured by glucose clamp with a glucose tracer. The liver is the major organ responsible for EGP, and thus suppression of EGP during glucose clamp is used as a marker of hepatic insulin sensitivity [12]. Similarly, the insulin-mediated anti-lipolytic effect is evaluated by suppression of circulating FFA concentrations during glucose clamp and is used as a marker of adipose tissue insulin sensitivity [15]. In this review, insulin resistance is defined as a decreased tissue response to insulin in each insulin target organ, including adipose tissue, muscle and liver, as mentioned above.

\section{Measurement of Intracellular Lipid Accumulation in Muscle and Liver}

Research on ectopic fat and insulin resistance in humans was greatly facilitated after the development of ${ }^{1} \mathrm{H}-\mathrm{MRS}$, a non-invasive means of separately measuring intramyocellular lipid (IMCL) and extramyocellular lipid (EMCL) [16]. Lipids in skeletal muscle exist in two forms: EMCL (adipose tissue) and IMCL. Because it has been suggested that IMCL, but not EMCL, impairs insulin signaling and induces insulin resistance in muscle, separate measurement of IMCL and EMCL can be used to evaluate the specific role of each lipid type on muscle insulin resistance. This is possible because ${ }^{1} \mathrm{H}-\mathrm{MRS}$ can distinguish EMCL and IMCL based on the position of the methylene signal, and the amount of lipid can be quantified by the signal intensity. This method is also applied to measure intrahepatic lipid (IHL), generally expressed as a percent value by using a water signal as an internal standard. This method is noninvasive and iterative, and compared with biopsy (e.g. 20 200 mg) it permits the measurement of intracellular lipids in much larger tissue volumes (e.g. $\left.1 \sim 2 \mathrm{~cm}^{3}\right)$. Thus, ${ }^{1} \mathrm{H}-\mathrm{MRS}$ measurement may reduce the sample bias resulting from the small size of tissue sample.

\section{Intracellular Lipid Accumulation and Insulin Resistance in Muscle}

By using the ${ }^{1} \mathrm{H}-\mathrm{MRS}$ method, an association between IMCL and insulin resistance was reported by 2 different groups in 1999. Krssak et al. [13] recruited normalweight, non-diabetic adults and measured IMCL and whole-body insulin sensitivity (MCR) by ${ }^{1} \mathrm{H}-\mathrm{MRS}$ and hyperinsulinemic euglycemic clamp, respectively. They demonstrated a negative correlation between IMCL accumulation and whole-body insulin sensitivity that was still significant even after adjustment for BMI, fasting plasma glucose, and age. On the other hand, Jacob et al. [14] recruited first-degree relatives of individuals with type 2 diabetes, and matched 13 lean, whole-body insulin-resistant subjects, determined by decreased MCR, and 13 lean, insulin-sensitive subjects in terms of sex, age, BMI, percent body fat, physical fitness, and waist-to-hip ratio. Then, they compared IMCL accumulation between the 2 groups and found that the insulinresistant subjects had $84 \%$ and $57 \%$ higher levels of IMCL in the soleus muscle (SOL) and tibialis anterior muscle (TA), respectively. Subsequently, Virkamäki et al. [17] divided 20 healthy men into high- and lowIMCL groups. They found that although BMI and fitness levels were comparable between the groups, the highIMCL group showed reduced whole-body insulin sensitivity and impaired insulin signaling at the levels of the insulin receptor (IR) and IR substrate-1-associated phosphatidylinositol 3-kinase. In these early studies, subjects were carefully selected to ensure that obesity did not have an effect on insulin sensitivity or IMCL accumulation. Therefore, they could suggest that IMCL accumulation is a mechanism of insulin resistance that is independent of obesity. 


\section{IMCL Accumulation, Circulating FFAs and Insulin Resistance}

It has been shown that acute FFA elevation by lipid infusion induces insulin resistance in peripheral tissues. Bachmann et al. demonstrated that elevation of circulating FFAs by lipid infusion for $6 \mathrm{~h}$ resulted in increases of $120.8 \%$ and $164.2 \%$ in IMCL levels in the SOL and TA, respectively. They also observed an approximately $40 \%$ reduction of whole-body insulin sensitivity (GIR) as determined by hyperinsulinemic euglycemic clamp. Finally, they found a significant correlation over time between GIR and IMCL levels during lipid infusion [4]. Similar IMCL changes after lipid infusion were also demonstrated by Boden et al. [18], who investigated the effects of 3 different protocols on IMCL changes. They infused insulin only, insulin with lipid or lipid only, and these 3 protocols achieved low, middle, and high levels of circulating FFAs, respectively. They found that IMCL levels were significantly increased after infusion of insulin with lipid and with lipid only, and there was a significant correlation between IMCL and elevated plasma FFA levels. Collectively, these data suggest that circulating FFA level is one of the determinants of IMCL accumulation. Considering the fact that circulating FFA elevation in obesity is associated with increased FFA release from adipose tissue [19], obesity may induce muscle insulin resistance through elevated FFAs and subsequent IMCL accumulation.

\section{Mitochondrial Function, IMCL Accumulation, and Insulin Resistance}

One of the key determinants of IMCL accumulation may be energy expenditure in myocytes, because the amount of stored IMCL is generally determined by the balance between FFA uptake and oxidation. Accordingly, Petersen et al. demonstrated impaired mitochondrial activity in elderly and insulin-resistant offspring of patients with type 2 diabetes [20, 21]. In addition, they found that impaired mitochondrial activity was closely associated with IMCL accumulation and peripheral insulin resistance. For example, they recruited young and elderly individuals matched for lean body mass and fat mass, and found that peripheral insulin sensitivity (Rd) was $40 \%$ lower in elderly subjects, and this difference was associated with a $45 \%$ increase in IMCL level and a $40 \%$ decrease in mitochondrial activity [20]. Similarly, in another study, insulin-resistant offspring of patients with type 2 diabetes were characterized by an $80 \%$ increase in IMCL level and a $30 \%$ decrease in mitochondrial activity [21]. These data suggest that impaired mitochondrial function may be a determinant of IMCL accumulation and muscle insulin resistance in elderly and insulin-resistant offspring of type 2 diabetes.

\section{Dietary Fat, Physical Activity, IMCL Accumulation, and Impaired Insulin Sensitivity}

IMCL levels are known to be affected by dietary fat and physical activity. In fact, high-fat (HF) diets were found to increase IMCL levels in healthy subjects [4, 22, $23]$ and in endurance runners $[22,24]$. We previously reported the effects of a eucaloric, HF, low-carbohydrate (E-HFLC) diet on 7 endurance runners and 7 sprinters. We found that the 3-day E-HFLC diet significantly increased IMCL levels in endurance runners, but not in sprinters [24]. Bachman et al. [4] also reported that a 3day HF diet increased IMCL levels in the TA and decreased whole-body insulin sensitivity (GIR). Interestingly, lipid infusion increased IMCL levels in all subjects, but there was a large interindividual variation in IMCL changes after the HF diet. This observation was consistent with our study, and we supposed that interindividual differences in the susceptibility to IMCL accumulation after fat loading may be partly explained by the type of exercise (e.g., aerobic or anaerobic). However, the determinants of susceptibility to IMCL accumulation following a HF diet are largely unknown.

To identify factors that increase the likelihood of IMCL accumulation after a HF diet, we recruited 50 nonobese men and provided a E-HFLC diet for 3 days [25, 26]. We observed significant increases in IMCL levels in the TA and SOL, and decreased muscle insulin sensitivity (GIR) [26]. The changes in IMCL level were negatively correlated with changes in GIR, suggesting that the former may be used as a marker to predict the latter. However, there were large interindividual variations in changes in both IMCL levels and insulin sensitivity. Thus, we searched for determinants of changes in IMCL levels after the E-HFLC diet. Our data suggested that low levels of high molecular weight (HMW) adiponectin and low daily physical activity with low fitness level were risk factors for post-diet IMCL accumulation [25]. In addition, mitochondrial content, evaluated by muscle biopsy, tended to negatively correlate with IMCL change [26]. In regard to these findings, adiponectin is secreted from adipocytes and increases AMP-activated protein kinase activity and promotes fat oxidation and mitochondrial biogenesis [27]. In addition, physical activity was shown to rapidly decrease IMCL levels in healthy subjects [22, 28]. Furthermore, 2-week calorie restriction with exercise (mainly brisk walking) decreased IMCL levels by $19 \%$ and increased peripheral insulin sensitivity (GIR) by $57 \%$ in patients with type 2 diabetes, 
whereas these changes were not observed under calorie restriction without exercise [29]. In addition, the changes in physical activity levels were negatively correlated with changes in IMCL levels [29].

Based on these results, we speculate that a HF diet, physical inactivity, lower fitness level, and the physiological effects of adiponectin and muscle mitochondria may together increase IMCL and impair insulin sensitivity in skeletal muscle independently of obesity. Thus, these factors may also contribute to insulin resistance and metabolic abnormalities in non-obese Asians.

\section{Insulin Resistance in Non-Obese Asians}

Although there have been numerous studies regarding insulin resistance in obese people, the number of studies which evaluated insulin resistance in non-obese Asians by hyperinsulinemic euglycemic clamp combined with a glucose tracer is very limited [12, 30-34]. In addition, when insulin sensitivity is measured in non-diabetic, non-obese subjects, infusion of lower amounts of insulin is necessary to separately evaluate insulin sensitivity in muscle and liver. Thus, we performed a 2-step (10 and $20 \mathrm{mU} / \mathrm{m}^{2}$ body surface area per minute) hyperinsulinemic euglycemic clamp study in 90 non-diabetic, nonobese (BMI $<25 \mathrm{~kg} / \mathrm{m}^{2}$ ) Japanese men, and focused particularly on those with a BMI between $23-25 \mathrm{~kg} / \mathrm{m}^{2}$ $(n=70)$ [12], which has been used as the definition for overweight in Asians [10]. In addition, subjects with metabolic syndrome and a BMI between $25-27.5 \mathrm{~kg} / \mathrm{m}^{2}$ (OB-MS) were also recruited as a positive control for obese Asians. This was a prospective observational study consisting of hypothesis-driven, hypothesis-generating research on the underlying mechanisms of metabolic abnormalities in non-obese subjects (Sportology Center Core Study) [12]. One of the main findings of the study was that impaired insulin sensitivity occurred in muscle (Rd), but not in liver (percent suppression of EGP by insulin at first step), in subjects with a BMI of 23-25 $\mathrm{kg} / \mathrm{m}^{2}$ (hereafter referred to as overweight subjects) who had as few as 1 cardiometabolic risk factor, while impaired insulin sensitivity in both muscle and liver was observed in OB-MS subjects. Insulin resistance in muscle was similar in the 2 groups. Correlation analysis revealed that decreased insulin sensitivity in muscle was well correlated with every measured cardiometabolic risk factor, including elevated fasting glucose, dyslipidemia, and hypertension, and the strongest correlation was with triglyceride levels $(r=-0.61)$. On the other hand, hepatic insulin sensitivity was significantly correlated with all parameters except high-density lipoprotein cholesterol, and the strongest correlation was with fasting plasma glucose $(r=-0.50)$. In addition, muscle insulin sensitiv- ity correlated with hepatic insulin sensitivity in the overweight subjects, but the association was weak $(r=0.38)$. These data suggest that among the overweight subjects, muscle insulin resistance, rather than hepatic insulin resistance, may have been the main pathogenic factor underlying metabolic abnormalities. Further correlation analysis indicated that muscle insulin sensitivity in overweight subjects was correlated with visceral fat area $(r=$ $-0.31)$, fitness level $(r=0.40)$, daily physical activity $(r$ $=0.25)$, HMW adiponectin $(r=0.48)$, protein intake $(r=$ $-0.29)$, fat intake $(r=-0.25)$, and C-reactive protein level $(r=-0.26)$. These data suggested that physical activity and dietary composition, as well as moderate adiposity and low-grade inflammation, may have been key determinants of muscle insulin resistance in overweight subjects. Interestingly, several of these factors were previously identified as determinants of IMCL accumulation after a E-HFLC diet [25], suggesting that they synergistically induce insulin resistance in skeletal muscle through IMCL accumulation. However, IMCL was not correlated with insulin resistance in this study. Because IMCL accumulation is also associated with high maximum oxygen uptake in insulin-sensitive subjects (the so-called athlete's paradox) [35], the high maximum oxygen uptake in healthy subjects may have affected the linear relationship between IMCL and muscle insulin sensitivity. In this regard, expression levels of plasma membrane-associated fatty acid-binding protein (FABPpm) in skeletal muscle were associated with the athlete's paradox seen in non-obese, healthy Japanese men [36].

Hepatic insulin sensitivity was not correlated with most of the factors we examined, but it was correlated with IHL $(r=-0.37)$ and protein intake $(r=-0.37)$, and was weakly but not significantly correlated with alcohol consumption ( $r=-0.22$ ). Because 1-week alcohol abstinence improves hepatic insulin sensitivity and fasting plasma glucose in non-obese Japanese men with mildly elevated fasting plasma glucose levels who consume alcohol [30], alcohol may be one of the factors that induces hepatic insulin resistance in non-obese Asians. High [37] or moderately high [38] alcohol consumption in Japanese was consistently shown to be associated with an increased risk of type 2 diabetes among lean Japanese men $\left(B M I \leq 22.0 \mathrm{~kg} / \mathrm{m}^{2}\right)$. A systematic review also suggested that moderate alcohol intake increased the risk of type 2 diabetes, especially in lean Japanese subjects [39].

\section{Investigating Metabolic Gradation in Non-Obese Asians}

Although several factors are associated with insulin resistance, causal relationships have not yet been demon- 
strated, and the etiology of insulin resistance in nonobese Asians is not fully understood. In particular, the following clinical question is very important in terms of metabolic disease prevention: when and how is insulin resistance induced in non-obese Asians? For example, type 2 diabetes commonly occurs in non-obese Asians, probably due to lower insulin secretion than in non-obese Caucasians. This suggests that Asians are metabolically fragile, because moderate insulin resistance can easily induce hyperglycemia due to lower insulin secretion. Detecting moderate insulin resistance at an early stage and identifying its etiology are required for effective metabolic disease prevention. Therefore, research on metabolic gradation, defined as the gradual transition from an insulin-sensitive to an insulin-resistant state, is of great interest, especially for identifying moderate early changes.

One possible early metabolic change is decreased insulin clearance and hyperinsulinemia [32, 40]. Insulin clearance is also evaluated by euglycemic hyperinsulinemic clamp, and the metabolic clearance rate of insulin (MCRI) is calculated by the ratio of the insulin infusion rate to the insulin level that is elevated by exogenous insulin [32]. In animal [41] and human [42] studies, intentional weight gain caused by a HF diet induced mild insulin resistance accompanied by hyperinsulinemia; of note, the major determinant of hyperinsulinemia was decreased insulin clearance rather than increased insulin secretion. Thus, impaired insulin clearance and hyperinsulinemia help maintain euglycemia against insulin resistance. However, several studies suggested that impaired insulin clearance and hyperinsulinemia also caused an insulin-resistant state and weight gain [43, 44]. The liver is the main organ responsible for insulin clearance, and carcinoembryonic antigen-related cell adhesion molecule 1 (CEACAM1) was found to promote receptor-mediated insulin uptake and degradation in hepatocytes [43, 44]. Liver-specific inactivation or global null-mutation of CEACAM1 impaired hepatic insulin clearance and induced chronic hyperinsulinemia, resulting in insulin resistance and adiposity [43, 44]. In addition, a HF diet diminished CEACAM1 expression and induced impaired insulin clearance, hyperinsulinemia, and insulin resistance in C57/BL6J mice; however, these effects were counteracted by liver-specific inducible CEACAM1 expression [44]. Further, lowering hyperinsulinemia prevented diet-induced obesity and insulin resistance in mice [45], and 40-h hyperinsulinemia (25$35 \mu \mathrm{U} / \mathrm{mL}$ ) induced insulin resistance in humans [46]. These data suggest that impaired insulin clearance and hyperinsulinemia could be the primary factors contributing to subsequent insulin-resistant states and weight gain. In this regard, we re-evaluated the Sportology Cen- ter Core Study database and investigated the significance of insulin clearance in non-obese, apparently healthy Japanese men [32]. The results suggested that subjects with low MCRI were characterized by moderate fat accumulation, low fitness level, hyperinsulinemia, and impaired insulin sensitivity in muscle but not in liver. In addition, the moderate insulin resistance in muscle seemed to be fully compensated for by elevated insulin levels secondary to decreased MCRI. Thus, impaired insulin clearance and hyperinsulinemia can occur in apparently healthy subjects without significant insulin resistance and may be a compensatory phenomenon that maintains glucose uptake and euglycemia in the face of moderate insulin resistance in muscle. In contrast to this beneficial effect, it is also possible that these changes may be an initial trigger of an insulin-resistant state and weight gain [43-46].

At least in animal models, impaired hepatic insulin clearance that is associated with decreased CEACAM1 expression in the liver may be a therapeutic target for the prevention of insulin resistance and obesity. However, the mechanisms of decreased insulin clearance in nonobese, healthy subjects are not yet fully understood. A correlation analysis revealed that fitness level was one of the determinants of insulin clearance. In another study, 1 year of aerobic exercise (jogging) enhanced muscle insulin sensitivity as well as insulin clearance in healthy young men [47], results that might have been due to increased expression of insulin-degrading enzymes in the liver and skeletal muscle [48]. Thus, exercise may simultaneously regulate both insulin sensitivity and insulin clearance, as well as balance the metabolic state.

Another question of interest is why slightly increased adiposity is associated with decreased insulin clearance in apparently healthy Japanese men. A previous study revealed that increasing adiposity was associated with a lower accumulation of subcutaneous fat and a higher accumulation of visceral fat in East Asians than in other ethnic groups, including white, black, Hispanic, and Southeast Asian [49]. This may be caused by insufficient insulin suppression of FFA release from adipose tissue, so-called adipose tissue insulin resistance $[50,51]$. Thus, it is hypothesized that adipose tissue insulin resistance is easily induced in East Asians by weight gain, leading to the reduced ability of subcutaneous adipose tissue to store lipids [52]. Lipid spillover, in the form of FFAs, may promote several metabolic changes such as visceral and ectopic fat accumulation, which can subsequently induce insulin resistance in muscle and liver [5, 50, 53]. On the other hand, FFA elevation decreases insulin clearance $[54,55]$, and thus adipose tissue insulin resistance may balance insulin resistance and insulin clearance and could therefore be another early change in the process 


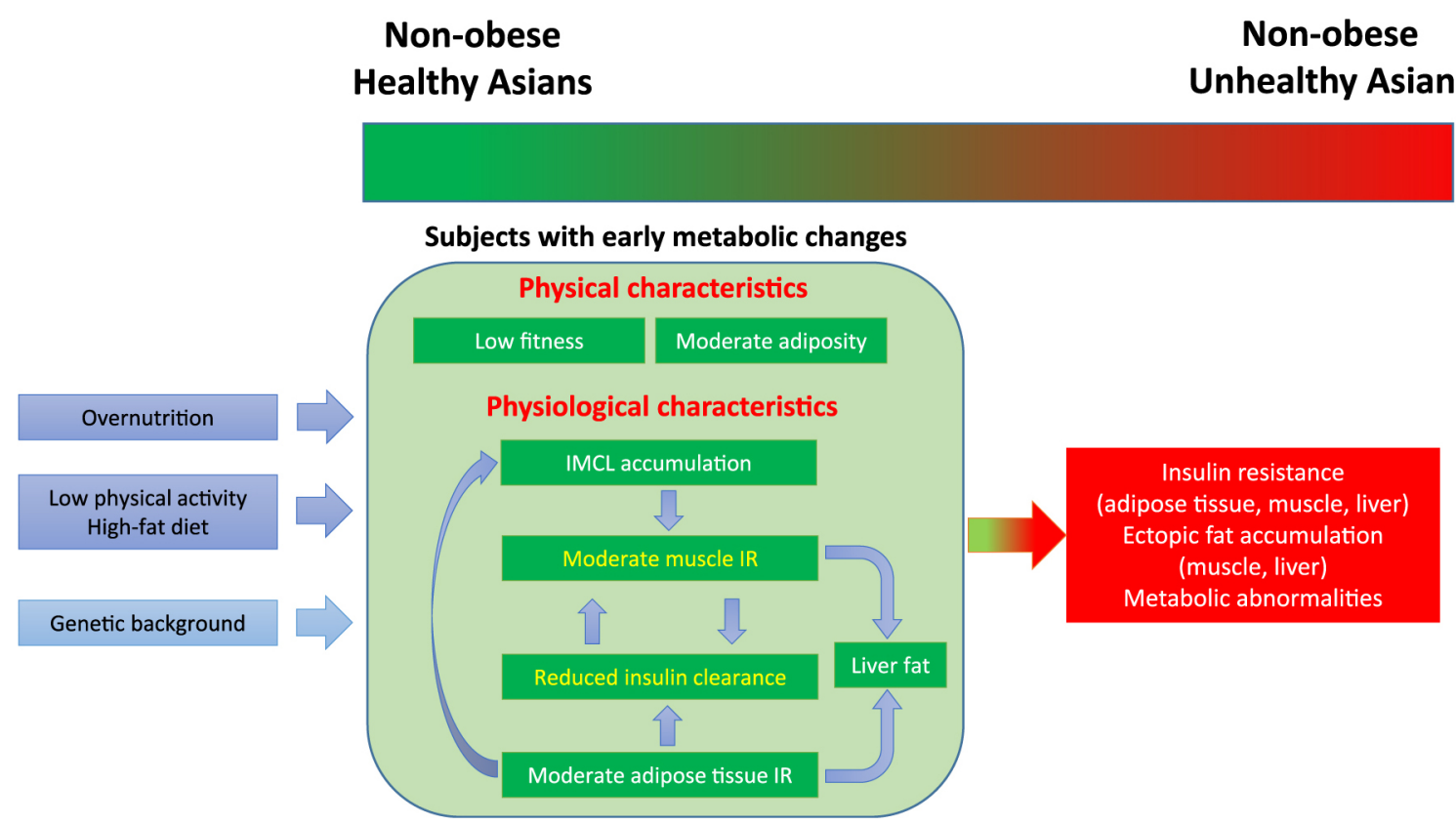

Fig. 1 Working hypothesis regarding the etiology of metabolic gradation and metabolic disease in non-obese Asians.

IMCL, intramyocellular lipid; IR, insulin resistance

leading to moderate insulin resistance. Our preliminary data suggested that even in non-obese, apparently healthy Japanese men, reduced adipose tissue insulin sensitivity was associated with moderate adiposity, moderate IHL accumulation, lower insulin clearance, and muscle insulin resistance [56].

A HF diet with low physical activity may be important for promoting insulin resistance in muscle. As mentioned above, a HF diet, physical inactivity, lower fitness level, and the effects of adiponectin and muscle mitochondria may together increase IMCL and impair insulin sensitivity in skeletal muscle independently of obesity $[25,26]$. In this regard, Bakker et al. [57] conducted a 5-day, HF, high-calorie diet intervention in young South Asian men and Caucasian men. Interestingly, they found that the HF, high-calorie diet impaired insulin sensitivity in muscle in South Asians but not in Caucasians. Thus, the effects of a HF diet on insulin sensitivity may occur more easily in Asians than in other ethnic groups.

Finally, the role of IHL accumulation in metabolic gradation is still unclear. Non-alcoholic fatty liver disease (NAFLD) is relatively common in Asian countries despite low BMI $[58,59]$, and fatty liver was identified as an independent risk factor for type 2 diabetes in a Japanese cohort, especially in subjects with low BMI [60]. It was demonstrated that FFAs released from adipose tissue were the main substrate for lipid accumulation in the liver in NAFLD patients [6], and adipose tissue insulin resistance was associated with fat accumulation and insulin resistance in the liver [61-63]. On the other hand, in non-diabetic, non-obese Japanese men [12] and nonobese Japanese with type 2 diabetes [31], IHL accumulation was closely associated with insulin resistance in muscle. As a result, it has been proposed that fatty liver is the consequence of muscle insulin resistance that alters the metabolic pathway of ingested carbohydrates away from skeletal muscle glycogen synthesis into de novo hepatic lipogenesis $[64,65]$. Thus, increased IHL accumulation may be a secondary effect of adipose tissue insulin resistance and muscle insulin resistance, and may exacerbate hepatic insulin resistance.

\section{Conclusions}

This review focused on the etiology of IMCL accumulation and muscle insulin resistance in East Asians, mainly with reference to our previous studies. Of note, we addressed metabolic gradation, especially the early metabolic changes involved. Because only a small number of studies have investigated the role of insulin resistance in non-obese East Asians by using glucose clamp with a tracer, it is difficult to formulate firm conclusions in this review. However, it is clear that moderate unfavorable metabolic changes arise during a much healthier state than was expected.

As shown in Fig. 1, several factors may contribute to early metabolic changes in non-obese, apparently healthy East Asians. For example, decreased physical activity and a HF diet might be important lifestyle changes contributing to the initiation of muscle insulin resistance in 
non-obese East Asians, because these 2 factors have been shown to increase IMCL and decrease muscle insulin sensitivity, especially in subjects with low fitness levels $[25,26]$. It is also possible that moderate adiposity may induce adipose tissue insulin resistance and elevate circulating FFAs, both of which subsequently increase IMCL and decrease insulin sensitivity in muscle [56]. Reduced adipose tissue insulin sensitivity may also contribute to decreased insulin clearance and hyperinsulinemia [56], which may be compensatory phenomena against moderate insulin resistance in muscle. Lipid accumulation in the liver may reflect insulin resistance in muscle and adipose tissues, because glucose and circulating FFAs are major sources of hepatic lipids in NAFLD patients. However, because hepatic insulin resistance is closely associated with glycemic control, that seen in non-diabetic subjects is moderate even if IHL is accumulated [12].

As shown in Fig. 1, even some non-obese, apparently healthy Japanese men had early metabolic changes; these individuals were characterized by low fitness levels and moderate adiposity as well as several physiological changes as described above $[32,56]$. These characteristics generally correlated with each other in this population. However, their causal relationships were not fully elucidated, and we suppose that these characteristics develop individually as a result of lifestyle changes and genetic predisposition, and together lead to early metabolic gradation despite an apparently healthy state. Importantly, impaired insulin clearance and hyperinsu- linemia could be initial triggers driving a subsequent insulin-resistant state and weight gain [43-46], thus leading to further progression of metabolic gradation. Further research is clearly required to clarify the pathogenesis of metabolic gradation and metabolic diseases in non-obese Asians, and these studies may ultimately be highly useful for promoting "Health Creation."

\section{Acknowledgments}

I thank Dr. Ryuzo Kawamori and Dr. Hirotaka Watada for their constant encouragement and support regarding my research. I also thank Dr. Yasushi Tanaka and Dr. Adria Giacca for their mentoring and collaboration. I finally want to express my sincere thanks to all colleagues who contributed to the research referred to in this review article.

\section{Disclosure Summary}

The author has nothing to disclose.

\section{Funding}

High Technology Research Center Grant, Strategic Research Foundation at Private Universities, Private University Research Branding Project and KAKENHI (23680069, 26282197 17K19929) from the Ministry of Education, Culture, Sports, Science and Technology of Japan.

\section{References}

1. Guilherme A, Virbasius JV, Puri V, Czech MP (2008) Adipocyte dysfunctions linking obesity to insulin resistance and type 2 diabetes. Nat Rev Mol Cell Biol 9: $367-$ 377.

2. Groop LC, Saloranta C, Shank M, Bonadonna RC, Ferrannini E, et al. (1991) The role of free fatty acid metabolism in the pathogenesis of insulin resistance in obesity and noninsulin-dependent diabetes mellitus. J Clin Endocrinol Metab 72: 96-107.

3. Ferrannini E, Barrett EJ, Bevilacqua S, DeFronzo RA (1983) Effect of fatty acids on glucose production and utilization in man. $J$ Clin Invest 72: 1737-1747.

4. Bachmann OP, Dahl DB, Brechtel K, Machann J, Haap $\mathrm{M}$, et al. (2001) Effects of intravenous and dietary lipid challenge on intramyocellular lipid content and the relation with insulin sensitivity in humans. Diabetes 50: 2579-2584.

5. Shulman GI (2014) Ectopic fat in insulin resistance, dyslipidemia, and cardiometabolic disease. $N$ Engl J Med 371: 1131-1141.
6. Donnelly KL, Smith CI, Schwarzenberg SJ, Jessurun J, Boldt MD, et al. (2005) Sources of fatty acids stored in liver and secreted via lipoproteins in patients with nonalcoholic fatty liver disease. $J$ Clin Invest 115: 1343-1351.

7. Chan JC, Malik V, Jia W, Kadowaki T, Yajnik CS, et al. (2009) Diabetes in Asia: epidemiology, risk factors, and pathophysiology. JAMA 301: 2129-2140.

8. International-Diabetes-Federation (2013) IDF Diabetes Atlas. 6th edition.

9. Pan WH, Yeh WT, Weng LC (2008) Epidemiology of metabolic syndrome in Asia. Asia Pac J Clin Nutr 17 Suppl 1: 37-42.

10. World-Health-Organization (2000) The Asia-Pacific perspective: redefining obesity and its treatment. Sydney: Health Communications Australia.

11. Groop LC, Bonadonna RC, DelPrato S, Ratheiser K, Zyck $\mathrm{K}$, et al. (1989) Glucose and free fatty acid metabolism in non-insulin-dependent diabetes mellitus. Evidence for multiple sites of insulin resistance. J Clin Invest 84: 205213. 
12. Takeno K, Tamura Y, Kawaguchi M, Kakehi S, Watanabe $\mathrm{T}$, et al. (2016) Relation between insulin sensitivity and metabolic abnormalities in Japanese men with BMI of 23$25 \mathrm{~kg} / \mathrm{m}^{2}$. J Clin Endocrinol Metab 101: 3676-3684.

13. Krssak M, Falk Petersen K, Dresner A, DiPietro L, Vogel SM, et al. (1999) Intramyocellular lipid concentrations are correlated with insulin sensitivity in humans: a $1 \mathrm{H}$ NMR spectroscopy study. Diabetologia 42: 113-116.

14. Jacob S, Machann J, Rett K, Brechtel K, Volk A, et al. (1999) Association of increased intramyocellular lipid content with insulin resistance in lean nondiabetic offspring of type 2 diabetic subjects. Diabetes 48: 11131119.

15. Ter Horst KW, van Galen KA, Gilijamse PW, Hartstra $\mathrm{AV}$, de Groot PF, et al. (2017) Methods for quantifying adipose tissue insulin resistance in overweight/obese humans. Int J Obes (Lond) 41: 1288-1294.

16. Szczepaniak LS, Babcock EE, Schick F, Dobbins RL, Garg A, et al. (1999) Measurement of intracellular triglyceride stores by $\mathrm{H}$ spectroscopy: validation in vivo. Am J Physiol 276: E977-E989.

17. Virkamaki A, Korsheninnikova E, Seppala-Lindroos A, Vehkavaara S, Goto T, et al. (2001) Intramyocellular lipid is associated with resistance to in vivo insulin actions on glucose uptake, antilipolysis, and early insulin signaling pathways in human skeletal muscle. Diabetes 50: 2337 2343.

18. Boden G, Lebed B, Schatz M, Homko C, Lemieux S (2001) Effects of acute changes of plasma free fatty acids on intramyocellular fat content and insulin resistance in healthy subjects. Diabetes 50: 1612-1617.

19. Mittendorfer B, Magkos F, Fabbrini E, Mohammed BS, Klein S (2009) Relationship between body fat mass and free fatty acid kinetics in men and women. Obesity (Silver Spring) 17: 1872-1877.

20. Petersen KF, Befroy D, Dufour S, Dziura J, Ariyan C, et al. (2003) Mitochondrial dysfunction in the elderly: possible role in insulin resistance. Science 300: 1140-1142.

21. Petersen KF, Dufour S, Befroy D, Garcia R, Shulman GI (2004) Impaired mitochondrial activity in the insulinresistant offspring of patients with type 2 diabetes. $N$ Engl J Med 350: 664-671.

22. Decombaz J, Schmitt B, Ith M, Decarli B, Diem P, et al. (2001) Postexercise fat intake repletes intramyocellular lipids but no faster in trained than in sedentary subjects. Am J Physiol Regul Integr Comp Physiol 281: R760R769.

23. Schrauwen-Hinderling VB, Kooi ME, Hesselink MK, Moonen-Kornips E, Schaart G, et al. (2005) Intramyocellular lipid content and molecular adaptations in response to a 1-week high-fat diet. Obes Res 13: 2088-2094.

24. Tamura $Y$, Watada H, Igarashi $Y$, Nomiyama T, Onishi T, et al. (2008) Short-term effects of dietary fat on intramyocellular lipid in sprinters and endurance runners. Metabolism 57: 373-379.

25. Sakurai Y, Tamura Y, Takeno K, Kumashiro N, Sato F, et al. (2011) Determinants of intramyocellular lipid accumu- lation after dietary fat loading in non-obese men. $J$ Diabetes Investig 2: 310-317.

26. Kakehi S, Tamura Y, Takeno K, Sakurai Y, Kawaguchi $\mathrm{M}$, et al. (2016) Increased intramyocellular lipid/impaired insulin sensitivity is associated with altered lipid metabolic genes in muscle of high responders to a high-fat diet. Am J Phyiol Endocrinol Metab 310: E32-E40.

27. Yamauchi T, Kamon J, Minokoshi Y, Ito Y, Waki H, et al. (2002) Adiponectin stimulates glucose utilization and fatty-acid oxidation by activating AMP-activated protein kinase. Nat Med 8: 1288-1295.

28. Larson-Meyer DE, Newcomer BR, Hunter GR (2002) Influence of endurance running and recovery diet on intramyocellular lipid content in women: a 1H NMR study. $\mathrm{Am}$ J Phyiol Endocrinol Metab 282: E95-E106.

29. Tamura Y, Tanaka Y, Sato F, Choi JB, Watada H, et al. (2005) Effects of diet and exercise on muscle and liver intracellular lipid contents and insulin sensitivity in type 2 diabetic patients. J Clin Endocrinol Metab 90: 3191-3196.

30. Funayama T, Tamura Y, Takeno K, Kawaguchi M, Kakehi $\mathrm{S}$, et al. (2017) Effects of alcohol abstinence on glucose metabolism in Japanese men with elevated fasting glucose: a pilot study. Sci Rep 7: 40277.

31. Furukawa Y, Tamura Y, Takeno K, Funayama T, Kaga H, et al. (2017) Impaired peripheral insulin sensitivity in nonobese Japanese patients with type 2 diabetes mellitus and fatty liver. J Diabetes Investig 9: 529-535.

32. Kaga H, Tamura Y, Takeno K, Kakehi S, Funayama T, et al. (2017) Correlates of insulin clearance in apparently healthy non-obese Japanese men. Sci Rep 7: 1462.

33. Chooi YC, Ding C, Chan Z, Choo J, Sadananthan SA, et al. (2018) Moderate weight loss improves body composition and metabolic function in metabolically unhealthy lean subjects. Obesity (Silver Spring) 26: 1000-1007.

34. Ding C, Chan Z, Chooi YC, Choo J, Sadananthan SA, et al. (2018) Regulation of glucose metabolism in nondiabetic, metabolically obese normal-weight Asians. $\mathrm{Am} \mathrm{J}$ Phyiol Endocrinol Metab 314: E494-E502.

35. Coen PM, Goodpaster BH (2012) Role of intramyocelluar lipids in human health. Trends Endocrinol Metab 23: 391398.

36. Kawaguchi M, Tamura Y, Kakehi S, Takeno K, Sakurai $\mathrm{Y}$, et al. (2014) Association between expression of FABPpm in skeletal muscle and insulin sensitivity in intramyocellular lipid-accumulated nonobese men. J Clin Endocrinol Metab 99: 3343-3352.

37. Tsumura K, Hayashi T, Suematsu C, Endo G, Fujii S, et al. (1999) Daily alcohol consumption and the risk of type 2 diabetes in Japanese men: the Osaka Health Survey. Diabetes Care 22: 1432-1437.

38. Waki K, Noda M, Sasaki S, Matsumura Y, Takahashi Y, et al. (2005) Alcohol consumption and other risk factors for self-reported diabetes among middle-aged Japanese: a population-based prospective study in the JPHC study cohort I. Diabet Med 22: 323-331.

39. Seike N, Noda M, Kadowaki T (2008) Alcohol consumption and risk of type 2 diabetes mellitus in Japanese: a sys- 
tematic review. Asia Pac J Clin Nutr 17: 545-551.

40. Watada H, Tamura Y (2017) Impaired insulin clearance as a cause rather than a consequence of insulin resistance. $J$ Diabetes Investig 8: 723-725.

41. Mittelman SD, Van Citters GW, Kim SP, Davis DA, Dea MK, et al. (2000) Longitudinal compensation for fatinduced insulin resistance includes reduced insulin clearance and enhanced beta-cell response. Diabetes 49: 21162125.

42. Erdmann J, Kallabis B, Oppel U, Sypchenko O, Wagenpfeil S, et al. (2008) Development of hyperinsulinemia and insulin resistance during the early stage of weight gain. Am J Phyiol Endocrinol Metab 294: E568-E575.

43. Poy MN, Yang Y, Rezaei K, Fernstrom MA, Lee AD, et al. (2002) CEACAM1 regulates insulin clearance in liver. Nat Genet 30: 270-276.

44. Al-Share QY, DeAngelis AM, Lester SG, Bowman TA, Ramakrishnan SK, et al. (2015) Forced hepatic overexpression of CEACAM1 curtails diet-induced insulin resistance. Diabetes 64: 2780-2790.

45. Mehran AE, Templeman NM, Brigidi GS, Lim GE, Chu $\mathrm{KY}$, et al. (2012) Hyperinsulinemia drives diet-induced obesity independently of brain insulin production. Cell Metab 16: 723-737.

46. Rizza RA, Mandarino LJ, Genest J, Baker BA, Gerich JE (1985) Production of insulin resistance by hyperinsulinaemia in man. Diabetologia 28: 70-75.

47. Oshida Y, Yamanouchi K, Hayamizu S, Sato Y (1989) Long-term mild jogging increases insulin action despite no influence on body mass index or VO2 max. $J$ Appl Physiol (1985) 66: 2206-2210.

48. Kurauti MA, Freitas-Dias R, Ferreira SM, Vettorazzi JF, Nardelli TR, et al. (2016) Acute exercise improves insulin clearance and increases the expression of insulindegrading enzyme in the liver and skeletal muscle of Swiss mice. PLoS One 11: e0160239.

49. Nazare JA, Smith JD, Borel AL, Haffner SM, Balkau B, et al. (2012) Ethnic influences on the relations between abdominal subcutaneous and visceral adiposity, liver fat, and cardiometabolic risk profile: the International Study of Prediction of Intra-Abdominal Adiposity and Its Relationship With Cardiometabolic Risk/Intra-Abdominal Adiposity. Am J Clin Nutr 96: 714-726.

50. Ebbert JO, Jensen MD (2013) Fat depots, free fatty acids, and dyslipidemia. Nutrients 5: 498-508.

51. Sondergaard E, Espinosa De Ycaza AE, Morgan-Bathke M, Jensen MD (2017) How to measure adipose tissue insulin sensitivity. J Clin Endocrinol Metab 102: 1193 1199.

52. Konige M, Wang H, Sztalryd C (2014) Role of adipose specific lipid droplet proteins in maintaining whole body energy homeostasis. Biochim Biophys Acta 1842: 393-
401.

53. Jensen MD (2008) Role of body fat distribution and the metabolic complications of obesity. J Clin Endocrinol Metab 93: S57-S63.

54. Wiesenthal SR, Sandhu H, McCall RH, Tchipashvili V, Yoshii H, et al. (1999) Free fatty acids impair hepatic insulin extraction in vivo. Diabetes 48: 766-774.

55. Hennes MM, Dua A, Kissebah AH (1997) Effects of free fatty acids and glucose on splanchnic insulin dynamics. Diabetes 46: 57-62.

56. Sugimoto D, Tamura Y, Takeno K, Kaga H, Someya Y, et al. (2018) Clinical significance of insulin sensitivity in adipose tissue in apparently healthy nonobese men. Diabetes 67.

57. Bakker LE, van Schinkel LD, Guigas B, Streefland TC, Jonker JT, et al. (2014) A 5-day high-fat, high-calorie diet impairs insulin sensitivity in healthy, young South Asian men but not in Caucasian men. Diabetes 63: 248-258.

58. Farrell GC, Wong VW, Chitturi S (2013) NAFLD in Asia -as common and important as in the West. Nat Rev Gastroenterol Hepatol 10: 307-318.

59. Azuma K, Kadowaki T, Cetinel C, Kadota A, El-Saed A, et al. (2009) Higher liver fat content among Japanese in Japan compared with non-Hispanic whites in the United States. Metabolism 58: 1200-1207.

60. Yamada T, Fukatsu M, Suzuki S, Wada T, Yoshida T, et al. (2010) Fatty liver predicts impaired fasting glucose and type 2 diabetes mellitus in Japanese undergoing a health checkup. J Gastroenterol Hepatol 25: 352-356.

61. Kotronen A, Juurinen L, Tiikkainen M, Vehkavaara S, Yki-Jarvinen H (2008) Increased liver fat, impaired insulin clearance, and hepatic and adipose tissue insulin resistance in type 2 diabetes. Gastroenterology 135: 122-130.

62. Kotronen A, Seppala-Lindroos A, Bergholm R, YkiJarvinen H (2008) Tissue specificity of insulin resistance in humans: fat in the liver rather than muscle is associated with features of the metabolic syndrome. Diabetologia 51: 130-138.

63. Bril F, Barb D, Portillo-Sanchez P, Biernacki D, Lomonaco R, et al. (2017) Metabolic and histological implications of intrahepatic triglyceride content in nonalcoholic fatty liver disease. Hepatology 65: 1132-1144.

64. Rabol R, Petersen KF, Dufour S, Flannery C, Shulman GI (2011) Reversal of muscle insulin resistance with exercise reduces postprandial hepatic de novo lipogenesis in insulin resistant individuals. Proc Natl Acad Sci U S A 108: 13705-13709.

65. Flannery C, Dufour S, Rabol R, Shulman GI, Petersen KF (2012) Skeletal muscle insulin resistance promotes increased hepatic de novo lipogenesis, hyperlipidemia, and hepatic steatosis in the elderly. Diabetes 61: 2711-2717. 